\title{
Subsets of T lymphocytes in the lesional skin of pityriasis rosea*
}

\author{
Shuqin Wang ${ }^{1,2}$, Liying $\mathrm{Fu}^{3}$, Wenhui $\mathrm{Du}^{1}$, Jun $\mathrm{Hu}^{2}$, Yongsheng $\mathrm{Zha}^{2}$, Peiguang Wang ${ }^{1}$
}

DOI: http:/ / dx.doi.org/10.1590/abd1806-4841.20197494

\begin{abstract}
BACKGROUND: Pityriasis rosea is a common papulosquamous disorder. However, its etiology and pathogenesis remain unclear.

Oвјестіve: We investigate the types of inflammatory cells infiltrating the lesional skin of pityriasis rosea and demonstrate whether T-cell-mediated immunity is involved in the pathogenesis of this condition or not.

MetHods: The biopsies were taken from the lesional skin of 35 cases of patients diagnosed with pityriasis rosea. The specimens were prepared in paraffin sections, then submitted to routine immunohistochemistry procedures using monoclonal antibodies directed against CD3, CD4, CD8, CD20 and CD45RO and horseradish peroxidase-labeled goat anti-human antibodies. The positive sections were determined by the ratio and staining intensity of positive inflammatory cells.

RESULTS: The mean score of positive CD3, CD4, CD8, and CD45RO staining was respectively 3.74 $\pm 3.88,5.67 \pm 4.40,2.94 \pm 3.42$ and $7.68 \pm 4.33$ in these pityriasis rosea patients $(\mathrm{P}<0.001)$. The percentage of positive staining was $54.29 \%(19 / 35), 69.7 \%(23 / 33)$, $40 \%(14 / 35)$ and $79.41 \%(27 / 34)(\mathrm{P}<0.05)$. However, the staining of CD20 was negative in all samples. The mean score of CD3 staining in patients with time for remission $\leq 60$ days $(4.90 \pm 4.21)$ was higher than that in patients with time for remission $>60$ days (2.00 \pm 2.5$)(\mathrm{P}<0.05)$, whereas no statistical difference in the mean score of CD4, CD8 and CD45RO staining was observed. StUDy Limitations: The sample size and the selected monoclonal antibody are limited, so the results reflect only part of the cellular immunity in the pathogenesis of pityriasis rosea.

CONCLUSION: Our findings support a predominantly T-cell mediated immunity in the development of pityriasis rosea.

Keywords: Immunity, cellular; Immunohistochemistry; Pityriasis rosea
\end{abstract}

\section{INTRODUCTION}

Pityriasis rosea (PR) is a common inflammatory papulosquamous disorder. The rash usually resolves within 6-8 weeks; however, the disease can last for a longer time in some patients. The persistence of disease is associated with the systemic reactivation of human herpes virus 6 (HHV-6) and human herpes virus 7 (HHV7). ${ }^{1}$ Although it is self-limited, this disorder has some unfavorable influences on most patients. Up to now, its etiology and pathogen- esis have not been well elucidated. Previously, we found the level of interferon- $\gamma$ was decreased in the sera of PR patients. ${ }^{2}$ There are very few studies about the immunohistochemical features of PR. Therefore, we performed immunohistochemical staining of the inflammatory cells infiltrating the skin lesions to demonstrate the role of T-cell-mediated immunity in the development of PR.

\footnotetext{
Received 31 July 2017.

Accepted 30 December 2017.

Financial support: None.

Conflict of interest: None.

Department of Dermatology, 1st Affiliated Hospital, Anhui Medical University, Hefei, China.

Department of Dermatology, the Anqing Municipal Hospital, Anqing, China.

Department of Dermatology, People's Hospital of Zhengzhou, Zhengzhou, China.
}

* Work conducted at the Key Laboratory of Dermatology, Ministry of Education, Anhui Medical University, Hefei, China.

E-mail: wpg2370@163.com 


\section{METHODS}

Thirty-five patients with PR (19 males and 16 females) were included in the study. Eight cases were of patients with $<1$ week duration, and 27 cases were of patients with $\geq 1$ week duration. The mean age of these patients was $29.06 \pm 9.43$ years (range $17-53$ years old). At the time of diagnosis, the mean duration of disease was 29.03 days (range 1-180 days). The mean time for remission of disease was $67.54 \pm 44.86$ days (range 21-240 days). Biopsies were taken from one of the secondary lesions after each patient gave their informed consent. All skin samples were fixed in $10 \%$ formalin, embedded in paraffin, then submitted to routine immunohistochemistry procedures using monoclonal antibody directed against CD3, CD4, CD8, CD20 and CD45RO and horseradish peroxidase-labeled goat anti-human antibodies. The positive sections were determined by the ratio and staining intensity of positive inflammatory cells. The score of staining intensity was rated 0 (negative), 1 (faint yellow), 2 (brown) and 3 (tan-colored), while the score of the ratio of positive cells was rated 0 (negative), 1 (<10\%), 2 (10\%-25\%), 3 (26\%-50\%), 4 (51\%-75\%) and 5 (75\%-100\%). The multiplication of these two scores is the final score. The staining of the section was considered positive if the final score was $\geq 3$. The mean score and the percentage of positive staining were compared for CD3, CD4, CD8 and CD45RO using analysis of variance and Pearson Chi-square test, respectively. The mean scores of CD3, CD4, CD8 and CD45RO staining was compared between two groups using Student's test. Number of Research Ethics Committee: 20180318.

\section{RESULTS}

The mean score for CD3, CD4, CD8, and CD45RO was $3.74 \pm 3.88,5.67 \pm 4.40,2.94 \pm 3.42$ and $7.68 \pm 4.33$ in these PR patients $(\mathrm{P}<0.001)$. The percentage of positive CD3, CD4, CD8, and CD45RO staining was, respectively, $54.29 \%$ (19/35 cases), 69.7\% (23/33 cases), $40 \%(14 / 35$ cases) and $79.41 \%(27 / 34$ cases) $(\mathrm{P}<0.05)$ (Table 1 and Figures 1, 2, 3, 4). However, the staining for CD20 was negative for all skin samples. There was no statistical difference in the mean score of CD3, CD4, CD8 and CD20 staining between the patients with duration of $\leq 1$ week and those with duration $>1$ week. The mean score for CD3 staining in patients with time for remission $\leq 60$ days $(4.90 \pm 4.21)$ was higher than that in the patients with time for remission $>60$ days $(2.00 \pm 2.5)(P<0.05)$, whereas there was no statistical difference in the mean score of CD4, CD8 and CD45RO staining (Table 2).

\begin{tabular}{|lccc|}
\hline \multicolumn{3}{|c|}{ TABLE 1: The mean score and the percentage of positive sam- } \\
ples for CD3, CD4, CD8 and CD45RO
\end{tabular}

${ }^{*} P$ value $<0.001 ;{ }^{* *} P$ value $<0.05$

\section{DISCUSSION}

Previous studies have suggested the association of viral infection and some drugs with the development of PR. ${ }^{3-7}$ The pathogenesis of PR remains unknown. In 2014, we investigated the levels of IL-2, interferon- $\gamma$, IL-4 and IL-10 in the sera of PR patients, and identified a reduced level of interferon- $\gamma$. Therefore, we proposed that weakened Th1 response is most likely to contribute to the pathogenesis of $\mathrm{PR} .{ }^{2}$ Hussein et $^{2} \mathrm{l}^{8}$ performed immunohistochemical stains for B-cells (CD20), T-cells (CD3), histiocytes (CD68) and T-cells with cytotoxic activity (granzyme-B) in PR and found significantly higher counts of immune cells in lesional skin compared to the normal skin. In the lesional skin, the immune cells were mainly CD3(+) T lymphocytes and CD68(+) cells (histiocytes). Neoh et al ${ }^{9}$ conducted immunochemical staining on 12 biopsy specimens taken from both herald patches and secondary patches of 6 PR patients. As a result, the dermal infiltrate of lymphocytes stained positively for monoclonal antibodies specific for T-cells, but there was lack of natural killer cell and B-cell activities in all samples. Moreover, the ratio

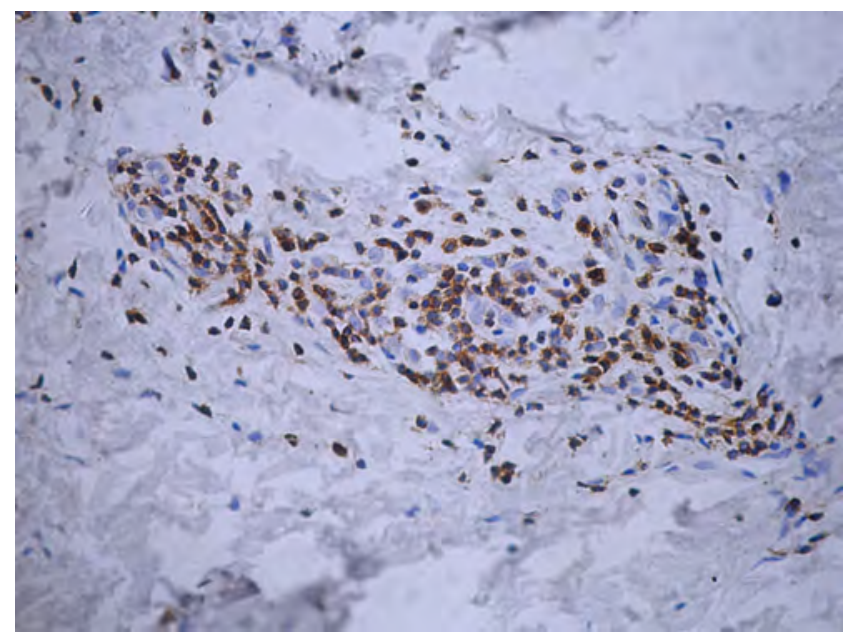

Figure 1: CD3 staining in the dermis (ABC-immunoperoxidase staining, $\times 400)$

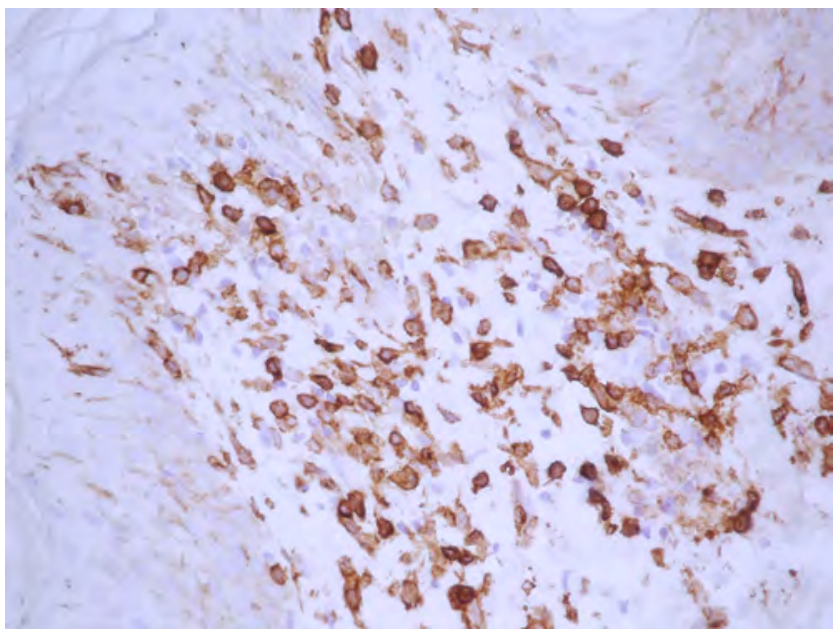

Figure 2: CD4 staining in the dermis (ABC-immunoperoxidase staining, $x 400)$ 


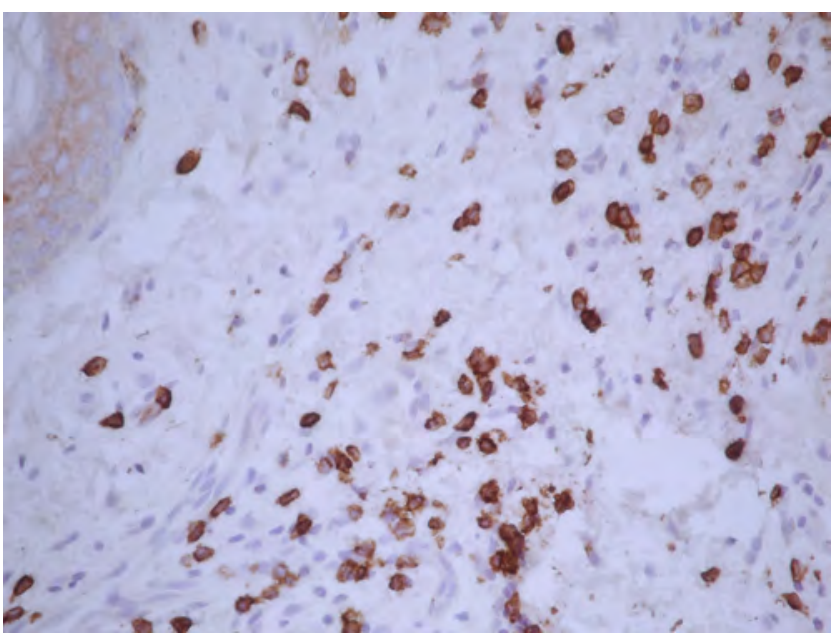

FIGURE 3: CD8 staining in the dermis (ABC-immunoperoxidase staining, $x 400)$

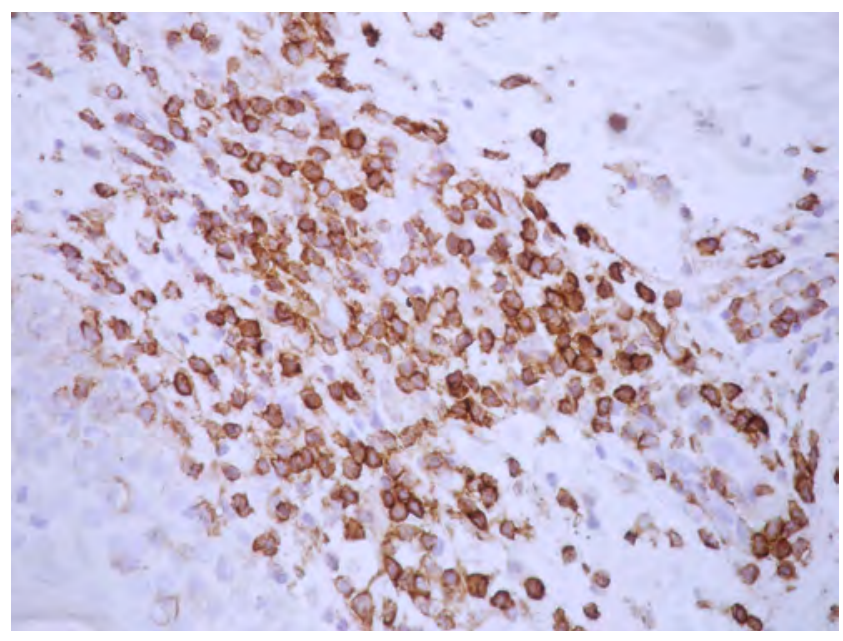

FIGURE 4: CD45RO staining in the dermis (ABC-immunoperoxidase staining, $\times 400)$

TABLE 2: The mean score of CD3, CD4, CD8 and CD45RO staining between groups with remission time $\leq 60$ days and group with remission time $>60$ days

\begin{tabular}{lcccccccc} 
Group & \multicolumn{2}{c}{ CD3 $^{*}$} & \multicolumn{2}{c}{ CD4 $^{* *}$} & \multicolumn{2}{c}{ CD8 $^{* *}$} & \multicolumn{2}{c}{ CD45RO $^{* *}$} \\
\cline { 2 - 8 } & $\mathbf{n}$ & score & $\mathbf{n}$ & score & n & score & n & score \\
\hline Remission time $\leq 60$ days & 21 & $4.90 \pm 4.21$ & 20 & $6.50 \pm 4.22$ & 21 & $3.33 \pm 3.47$ & 20 & $8.35 \pm 3.73$ \\
Remission time >60days & 14 & $2.00 \pm 2.57$ & 13 & $4.38 \pm 4.52$ & 14 & $2.36 \pm 3.39$ & 14 & $6.71 \pm 5.04$ \\
\hline
\end{tabular}

${ }^{*} P$ value $<0.05 ; * *$ value $>0.05$

of CD4(+) /CD8 (+) T-cells in the dermal infiltrate was increased in most specimens.

In this study, the results revealed that inflammatory cells with positive $\mathrm{CD} 3, \mathrm{CD} 4, \mathrm{CD} 8$ and $\mathrm{CD} 45 \mathrm{RO}$ staining predominated in the skin lesions of PR patients. The mean score of CD45RO staining was significantly higher than that of CD3, CD4 and CD8 staining $(\mathrm{P}<0.001)$. In addition, 27 of $34(79.41 \%)$ patients showed positive CD45RO staining, 19/35 (54.29\%) positive CD3 staining, 23/33 (69.7\%) positive CD4 staining and 14/35 (40\%) positive CD8 staining $(\mathrm{P}<0.05)$. However, all patients were negative for $\mathrm{CD} 20$ staining. These findings indicate that T-cells rather than B-cells play an important role in the development of PR. We compared the mean scores of CD3, CD4, CD8 and CD45RO staining between patients with $\leq 1$ week duration and patients with $>1$ week duration, and between the patients with time for remission $\leq 60$ days and the patients with time for remission $>60$ days. Patients with time for remission $\leq 60$ days had the higher mean score of CD3 staining than the patients with time for remission $>60$ days.

\section{CONCLUSION}

Our findings support that a predominantly T-cell-mediated immunity participated in the development of PR. $\square$ 


\section{REFERENCES}

1. Drago F, Broccolo F, Ciccarese G, Rebora A, Parodi A. Persistent pityriasis rosea: an unusual form of pityriasis rosea with persistent active HHV-6 and HHV-7 infection. Dermatology. 2015;230:23-6.

2. Zeng M, Zhao SX, Liu LH, Zuo XB, Zheng XD, Li T, et al. Decreased Serum Level of Interferon-gamma in Patients with Pityriasis Rosea. Ann Dermatol. 2014:26:522-3.

3. Broccolo F, Drago F, Careddu AM, Foglieni C, Turbino L, Cocuzza CE, et al. Additional evidence that pityriasis rosea is associated with reactivation of human herpesvirus-6 and -7. J Invest Dermatol. 2005;124:1234-40.

4. Drago F, Broccolo F, Rebora A. Pityriasis rosea: an update with a critical appraisal of its possible herpesviral etiology. J Am Acad Dermatol. 2009;61:303-18.

5. Brazzelli V, Prestinari F, Roveda E, Barbagallo T, Bellani E, Vassallo C, et al. Pityriasis rosea-like eruption during treatment with imatinib mesylate: description of 3 cases. J Am Acad Dermatol. 2005;53:S240-3.
6. Rajpara SN, Ormerod AD, Gallaway L. Adalimumab-induced pityriasis rosea. J Eur Acad Dermatol Venereol. 2007;21:1294-6.

7. Guarneri C, Polimeni G, Nunnari G. Pityriasis rosea during etanercept therapy. Eur Rev Med Pharmacol Sci. 2009;13:383-7.

8. Hussein MR, Abdel-Magid WM, Saleh R, Nada E. Phenotypical characteristics of the immune cells in allergic contact dermatitis, atopic dermatitis and pityriasis rosea. Pathol Oncol Res. 2009:15:73-9.

9. Neoh CY, Tan AW, Mohamed K, Sun YJ, Tan SH. Characterization of the inflammatory cell infiltrate in herald patches and fully developed eruptions of pityriasis rosea. Clin Exp Dermatol. 2010;35:300-4.

\section{AUTHORS'CONTRIBUTIONS}

$\begin{array}{ll}\text { Shuqin Wang } & \text { (D) ORCID 0000-0002-0176-6599 }\end{array}$

Statistical analysis; Elaboration and writing of the manuscript; Obtaining, analyzing and interpreting the data; Intellectual participation in propaedeutic and/or therapeutic conduct of the cases studied; Critical review of the literature; Critical review of the manuscript
Liying Fu
iD ORCID 0000-0001-7069-8676

Conception and planning of the study, Intellectual participation in propaedeutic and/or therapeutic conduct of the cases studied

Wenhui Du

(iD) ORCID 0000-0001-6474-703X

Obtaining, analyzing and interpreting the data; Intellectual participation in propaedeutic and/or therapeutic conduct of the cases studied

Jun $\mathrm{Hu}$

iD ORCID 0000-0002-9571-0667

Obtaining, analyzing and interpreting the data

Yongsheng Zha

(D) ORCID 0000-0002-0831-4851

Obtaining, analyzing and interpreting the data

Peiguang Wang

ORCID 0000-0002-3384-3587

Statistical analysis; Approval of the final version of the manuscript; Conception and planning of the study; Obtaining, analyzing and interpreting the data; Effective participation in research orientation; Intellectual participation in propaedeutic and/or therapeutic conduct of the cases studied; Critical review of the literature; Critical review of the manuscript

How to cite this article: Wang S, Fu L, Du W, Hu J, Zha Y, Wang P. Subsets of T lymphocytes in the lesional skin of pityriasis rosea. An Bras Dermatol. 2019;94(1):52-5. 